\title{
Visual Inspection Methods as Screening Test for Cervical Cancer in Low-resource Settings
}

\author{
${ }^{1}$ Soni Gupta, ${ }^{2}$ Amit Kyal, ${ }^{3}$ Partha Mukhopadhyay
}

\section{ABSTRACT}

Aim: The study aims to assess the utility of visual inspection methods-visual inspection with acetic acid (VIA) and visual inspection with Lugol's iodine (VILI)_for detecting premalignant lesions of the cervix in low-resource settings.

Materials and methods: The duration of the study was 1 year from May 1, 2014 to April 31, 2015 and was conducted at Medical College, Kolkata. A total of 2,278 apparently healthy, sexually active women aged 21 to 45 years were selected for the study. A Pap smear, VIA, and VILI were performed on all patients. Colposcopy-guided biopsy (considered as gold standard investigation) was performed on patients who tested positive for any of the above screening tests. The sensitivity, specificity, positive predictive value (PPV), and negative predictive value (NPV) of the various screening tests were compared.

Results: A total of 2,278 women were screened, of which 382 were lost to follow-up. Of the remaining 1,896 women, positive screening test was reported with Pap smear for 162 (8.5\%), VIA for $306(16.08 \%)$, and VILI for $338(17.83 \%)$. In the present study, the sensitivity, specificity, PPV, and NPV of Pap smear were $81.36,93.79,29.63$ and $99.37 \%$ respectively. For VIA, sensitivity, specificity, PPV, and NPV were $86.75,86.06,16.34$, and $99.43 \%$, whereas for VILI were $86.44,84.38,15.09$, and $99.49 \%$ respectively. Although the specificity and PPV of Pap smear were slightly higher than the visual inspection methods, there was no significant difference in the sensitivities and the NPV.

Conclusion: The sensitivity and specificity of VIA and VILI are comparable to cytology and do not require any cost-intensive paraphernalia. They can be easily done and interpreted by trained health workers, reducing the burden on tertiary care hospitals. Visual inspection methods like VIA and VILI are the most legitimate alternatives to cytology for screening cervical cancer, especially in low-resource settings.

Keywords: Colposcopy, Cross-sectional study, Low-resource settings, Pap smear, Screening, Visual inspection with acetic acid, Visual inspection with Lugol's iodine.

How to cite this article: Gupta S, Kyal A, Mukhopadhyay P. Visual Inspection Methods as Screening Test for Cervical Cancer in Low-resource Settings. J South Asian Feder Obst Gynae 2018;10(2):88-91.

\footnotetext{
${ }^{1}$ Postgraduate Trainee, ${ }^{2}$ Associate Professor, ${ }^{3}$ Professor and Head

${ }^{1-3}$ Department of Obstetrics and Gynecology, Medical College and Hospital, Kolkata, West Bengal, India

Corresponding Author: Amit Kyal, Regent Court, Block 2 Flat-5D, VIP Road, Kolkata, West Bengal, India, Phone: +919339108591, e-mail: amitkyal@yahoo.com
}

Source of support: Nil

Conflict of interest: None

Date of received: 15 March 2018

Date of acceptance: 20 April 2018

Date of publication: July 2018

\section{INTRODUCTION}

Cervical cancer is the fourth most frequently diagnosed malignancy in women worldwide. In India, every year, 122,844 new cases are being detected, making it the second most common cancer in women aged 15 to 44 years. ${ }^{1}$ When viewed from a global perspective, these figures account for approximately $25 \%(67,500)$ of cervical cancer deaths. ${ }^{2}$ It is projected that by 2030 , almost half a million women will die of cervical cancer, with over $98 \%$ of these deaths expected to occur in low- and middleincome countries. ${ }^{3}$ In resource-constrained settings, the burden of cervical cancer weighs heavily, largely as a result of lack of screening programs. ${ }^{4}$

It has long been thought that the Pap smear was the "ideal" screening tool, but several studies have shown that the sensitivity of conventional cytology is limited..$^{5-7}$ In underresourced regions, cytology-based screening is likely to face operational hurdles. At this moment, no single screening method exists that is devoid of pitfalls. Newer methodologies, developed to increase the sensitivity, also increase the cost. As visual screening tests are simple, affordable, acceptable, feasible, and reasonably accurate, they can be readily used in a variety of health care settings in both developing and developed countries. ${ }^{8-10}$

Researchers have urged that India immediately needs effective policies to reduce the country's huge burden of cervical cancer. The first step would be to identify a screening modality that is effective, with good sensitivity, specificity, high predictive value, feasibility for broad use, and acceptability in terms of cost, risk, and patient tolerability. ${ }^{11}$ With this background, the current study aims to analyze visual screening methods as a screening modality for cervical cancer in low-resource settings.

\section{MATERIALS AND METHODS}

The study was conducted between May 1, 2014 and April 31, 2015 in the outpatient department (OPD) and 
Visual Inspection Methods as Screening Test for Cervical Cancer

indoor ward, Department of Obstetrics and Gynecology, Medical College Kolkata. Apparently healthy, ambulant, sexually active women aged 21 to 45 years with an intact uterus were selected for the study.

Women who were never sexually active, were pregnant, having active bleeding per vaginum, having frank growth on the cervix, having prior treatment for cervical intraepithelial neoplasia (CIN) or posthysterectomy were excluded from the study.

This was an observational cross-sectional study using complete enumeration method. A total of 2,278 women were enrolled in the study after institutional ethics approval and consent from the study subjects.

In all cases, a thorough history and a complete physical and gynecological examination were done. Firstly, a Pap smear was taken with Ayre's spatula and endocervical brush and was evaluated by standard protocols in the first sitting in the OPD. Following the Pap smear in another sitting in minor operation theater, VIA was performed with 5\% acetic acid and results noted after 1 minute. Subsequently Lugol's iodine was applied. Colposcopic-guided biopsy (considered as gold standard investigation/reference test) was performed for patients who had at least one abnormal screening test.

Pap smear was considered positive if the report indicated atypical squamous cells of undetermined significance and above. Dense white lesion with sharp border seen in the transformation zone with one border abutting the squamocolumnar junction was considered as VIA positive; VILI positive was any dense, thick, bright, mustard-yellow noniodine uptake areas seen in the transformation zone, close to or abutting the squamocolumnar junction.

A diagnosis of CIN2+ (CIN2, CIN3, and invasive carcinoma) on histology was considered as true positive. Collected data were statistically analyzed to determine sensitivity, specificity, PPV, and NPV of Pap smear, VIA, and VILI.

\section{RESULTS}

A total of 2,278 women were screened, of which 382 were lost to follow-up and an effective sample size of 1,896 was used for statistics. Of the 1,896 women, positive screening test was reported with Pap smear for 162 (8.5\%), VIA for $306(16.14 \%)$, and VILI for $338(17.83 \%)$. A total of $59(3.1 \%)$ patients had CIN2+. The detection rates of CIN2 + by Pap smear were $2.53 \%$, VIA $2.63 \%$, and VILI $2.68 \%$ (Tables 1 to 6 ).

\section{DISCUSSION}

Cervical cancer is the most common genital cancer in Indian women. Morbidity and mortality can be decreased
Table 1: Screening method and number of women who tested positive

\begin{tabular}{llll}
\hline $\begin{array}{l}\text { Screening } \\
\text { method }\end{array}$ & $\begin{array}{l}\text { Number } \\
\text { screened }\end{array}$ & Positive & Percentage \\
\hline Pap smear & 1,896 & 162 & 8.50 \\
VIA & 1,896 & 306 & 16.14 \\
VILI & 1,896 & 338 & 17.83 \\
\hline
\end{tabular}

Table 2: Distribution of reference diagnosis on histopathological examination

\begin{tabular}{lll}
\hline Reference diagnosis & Total & Percentage \\
\hline Normal/inflammation & 1,669 & 88.02 \\
CIN1 & 168 & 8.86 \\
CIN2 & 43 & 2.27 \\
CIN3 & 14 & 0.74 \\
Invasive cancer & 2 & 0.11 \\
\hline Total & 1,896 & 100 \\
\hline
\end{tabular}

Table 3: Distribution of reference diagnosis by screening test results

\begin{tabular}{llll}
\hline Screening test & $\begin{array}{l}\text { Reference test CIN2+ normal/ } \\
\text { inflammation/atypia/CIN1 }\end{array}$ & Total \\
\hline $\begin{array}{l}\text { Pap smear } \\
\text { Positive }\end{array}$ & 48 & 114 & 162 \\
Negative & 11 & 1,723 & 1,734 \\
VIA & & & \\
Positive & 50 & 256 & 306 \\
Negative & 9 & 1,581 & 1,590 \\
VILI & & & \\
Positive & 51 & 287 & 338 \\
Negative & 8 & 1,550 & 1,558 \\
\hline
\end{tabular}

Table 4: Test characteristics of Pap smear in detecting CIN2+ lesions

\begin{tabular}{lll}
\hline Statistic & Value (\%) & $95 \% \mathrm{Cl}$ \\
\hline Sensitivity & 81.36 & $69.09-90.31 \%$ \\
Specificity & 93.79 & $92.59-94.85 \%$ \\
PPV & 29.63 & $22.72-37.30 \%$ \\
NPV & 99.37 & $98.87-99.68 \%$ \\
\hline
\end{tabular}

$\mathrm{Cl}$ : Confidence interval

Table 5: Test characteristics of VIA in detecting CIN2+ lesion

\begin{tabular}{lll}
\hline Statistic & Value (\%) & $95 \% \mathrm{Cl}$ \\
\hline Sensitivity & 84.75 & $73.01-92.78 \%$ \\
Specificity & 86.06 & $84.40-87.62 \%$ \\
PPV & 16.34 & $12.38-20.97 \%$ \\
NPV & 99.43 & $98.93-99.74 \%$ \\
\hline
\end{tabular}

$\mathrm{Cl}$ : Confidence interval

Table 6: Test characteristics of VILI in detecting CIN2+ lesions

\begin{tabular}{lll}
\hline Statistic & Value (\%) & $95 \% \mathrm{Cl}$ \\
\hline Sensitivity & 86.44 & $75.02-93.96 \%$ \\
Specificity & 84.38 & $82.63-86.01 \%$ \\
PPV & 15.09 & $11.45-19.36 \%$ \\
NPV & 99.49 & $98.99-99.78 \%$ \\
\hline
\end{tabular}

$\mathrm{Cl}$ : Confidence interval 
if it is detected in its preinvasive state. Despite this, India lacks a national program for the prevention of cervical cancer. A mass screening program similar to the ones used in developed countries does not seem to be feasible in a developing country like India.

In the International Agency for Research on Cancer multicenter study done in India and Africa by Sankaranarayanan et $\mathrm{al}^{8} 16.1 \%$ were VIA positive, $16.4 \%$ were VILI positive, and 1,063 were diagnosed with high-grade squamous intraepithelial lesion.

Basu et $\mathrm{al}^{9}$ conducted a study titled "Visual inspection with acetic acid and cytology in the early detection of cervical neoplasia in Kolkata, India." All screened women were evaluated by colposcopy and biopsies were directed in those with colposcopic abnormalities; 18.7, 17.7 and $8.2 \%$ of the women were tested positive for VIA, VIAM, and cytology respectively; 122 women had a final diagnosis of CIN2-3 lesions.

The prevalence of CIN2+ lesions in the present study population was found to be $3.1 \%$. Of the 1,896 women, positive screening test was reported with Pap smear for 162 (8.5\%), VIA for 306 (16.14\%), and VILI for 338 (17.83). A total of $59(3.1 \%)$ patients had CIN2+. The detection rates of CIN2+ by Pap smear were 2.53\%, VIA 2.63\%, and VILI 2.68\%.

The sensitivity, specificity, PPV, and NPV of Pap smear cytology in our study were 81.36, 93.79, 29.63, and $99.37 \%$ respectively. Pap smear was easy to perform but was relatively costly and the results were delayed due to dependence on the laboratory for interpretation. This was also the group which was maximally lost to follow-up.

In the present study, the sensitivity, specificity, PPV, and NPV of VIA were 86.75, 86.06, 16.34 and 99.43\%, whereas the sensitivity, specificity, PPV, and NPV of VILI were 86.44, 84.38, 15.09 and $99.49 \%$ respectively. The VIA and VILI were found to be easy to perform and to interpret. They were technically less taxing, low cost, and acceptable to the patient with no alarming adverse effects. The results could be quoted immediately which made planning for the next course of action easier.

The equipment required for colposcopy was found to be costly and the learning curve was found to be more tedious and prolonged than both cytology and visual inspection methods, although once fully acquainted with the methodology and interpretation it gave the best results. Also colposcopes are nonportable, which makes it an unlikely candidate for mass screening.

Although the specificity and PPV of Pap smear were slightly higher than the visual inspection methods, there was no significant difference in the sensitivities and the NPV.
In the present study, the results are comparable with that of multiple studies, suggesting that VIA and VILI should find a place as an alternative low-cost method of screening and case finding for cervical neoplasia.

All women were advised proper follow-up. Women with CIN2 and above were appropriately treated at our hospital.

\section{CONCLUSION}

Mass screening of cervical cancer in our country is an issue that needs to be addressed urgently. Cytology is expensive as it requires sophisticated laboratory equipment coupled with the fact that the results can be considerably delayed. A screening program with a wide outreach is the need of the hour for low-resource settings in a developing country. The sensitivity and specificity of VIA and VILI are comparable to cytology and do not require any cost-intensive paraphernalia. They can be easily done and interpreted by trained health workers, reducing the burden on tertiary care hospitals. Visual inspection methods like VIA and VILI are the most legitimate alternatives to cytology for screening cervical cancer.

\section{REFERENCES}

1. Ferlay J, Soerjomataram I, Ervik M, Dikshit R, Eser S, Mathers C, Rebelo M, Parkin DM, Forman D, Bray, F. GLOBOCAN 2012 v1.0, Cancer Incidence and Mortality Worldwide: IARC Cancer Base No. 11. Lyon, France: International Agency for Research on Cancer; 2013.

2. American Cancer Society. Global cancer facts and figures. 3rd ed. Atlanta: American Cancer Society; 2015.

3. Bhaumik S. India has world's highest number of cervical cancer deaths. BMJ 2013 May 13;346:f3108.

4. Parkin DM. Screening for cervix cancer in developing countries. In: Miller AB, Chamberlain J, Day NE, Hakama M, Prorok PC, editors. Cancer screening. Cambridge: Cambridge University Press; 1991. pp. 184-198.

5. Fahey MT, Irwig L, Macaskill P. Meta-analysis of Pap test accuracy. Am J Epidemiol 1995 Apr;141(7):680-689.

6. Nanda K, McCrory DC, Myers ER, Bastian LA, Hasselblad V, Hickey JD, Matchar DB. Accuracy of the Papanicolaou test in screening for and follow-up of cervical cytologic abnormalities: a systematic review. Ann Intern Med 2000 May; 132(10):810-819.

7. Sankaranarayanan R, Thara S, Sharma A, Roy C, Shastri S, Mahe C, Muwonge R, Fontanière B, Multicentre Study Group on Cervical Cancer Early Detection in India. Accuracy of conventional cytology: results from a multicentre screening study in India. J Med Screen 2004;11(2):77-84.

8. Sankaranarayanan R, Basu P, Wesley RS, Mahe C, Keita N, Mbalawa CC, Sharma R, Dolo A, Shastri SS, Nacoulma M, et al. Accuracy of visual screening for cervical neoplasia: results from an IARC multicentre study in India and Africa. Int J Cancer 2004 Jul 20;110(6):907-913.

9. Basu PS, Sankaranarayanan R, Mandal R, Roy C, Das P, Choudhury D, Bhattacharya D, Chatterjee R, Dutta K, Barik S, 
et al. Visual inspection with acetic acid and cytology in the early detection of cervical neoplasia in Kolkata, India. Int J Gynecol Cancer 2003 Sep-Oct;13(5):626-632.

10. Poli UR, Bidinger PD, Gowrishankar S. Visual Inspection with Acetic Acid (VIA) screening program: 7 years experience in early detection of cervical cancer and pre-cancers in rural south India. Indian J Community Med 2015 Jul-Sep;40(3): 203-207.

11. Denny L, Kuhn L, De Souza M, Pollack AE, Dupree W, Wright TC Jr. Screen-and-treat approaches for cervical cancer prevention in low-resource settings: a randomized controlled trial. JAMA 2005 Nov;294(17):2173-2181. 